Material behaviour

\title{
Thermal and hydrolytic degradation of electrospun fish gelatin membranes
}

\author{
D.M. Correia ${ }^{\mathrm{a}, \mathrm{b}}$, J. Padrão ${ }^{c}$, L.R. Rodrigues ${ }^{c}$, F. Dourado ${ }^{c}$, S. Lanceros-Méndez ${ }^{\mathrm{b}}$, \\ V. Sencadas ${ }^{\mathrm{b}, \mathrm{d}, *}$

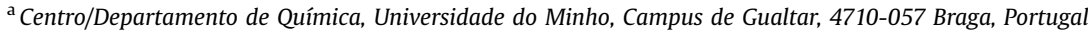 \\ ${ }^{\mathrm{b}}$ Centro/Departamento de Física da Universidade do Minho, Campus de Gualtar, 4710-057 Braga, Portugal \\ ${ }^{\mathrm{c}}$ Institute for Biotechnology and Bioengineering, Centre for Biological Engineering, University of Minho, Campus Gualtar, 4710-057 Braga, \\ Portugal \\ d Escola Superior de Tecnologia, Instituto Politécnico do Cávado e do Ave, Campus do IPCA, 4750-810, Barcelos, Portugal
}

\section{A R T I C L E I N F O}

\section{Article history:}

Received 3 April 2013

Accepted 11 May 2013

\section{Keywords:}

Fish gelatin

Electrospun membranes

Thermal degradation

Hydrolytic degradation

Biomaterials

\begin{abstract}
A B S T R A C T
The thermal and hydrolytic degradation of electrospun gelatin membranes cross-linked with glutaraldehyde in vapor phase has been studied. In vitro degradation of gelatin membranes was evaluated in phosphate buffer saline solution at $37^{\circ} \mathrm{C}$. After 15 days under these conditions, a weight loss of $68 \%$ was observed, attributed to solvation and depolymerization of the main polymeric chains. Thermal degradation kinetics of the gelatin raw material and as-spun electrospun membranes showed that the electrospinning processing conditions do not influence polymer degradation. However, for cross-linked samples a decrease in the activation energy was observed, associated with the effect of glutaraldehyde cross-linking reaction in the inter- and intra-molecular hydrogen bonds of the protein. It is also shown that the electrospinning process does not affect the formation of the helical structure of gelatin chains.
\end{abstract}

(c) 2013 Elsevier Ltd. All rights reserved.

\section{Introduction}

Gelatin is a biodegradable, biocompatible, non-toxic and non-carcinogenic biopolymer [1]. It is typically obtained through partial denaturation of collagen and accounts for $30 \%$ of the total animal protein in all animals. Collagen comprises all 20 amino acids in its three $\alpha$-chains, which are stabilized and interlaced by hydrogen bonds into a triple helix that rotates clockwise [2-4]. Collagen may be partially degraded using two distinct pre-treatments, the acid and the alkali, resulting in type-A and type-B gelatin, respectively [3,5]. Gelatin possesses some drawbacks

\footnotetext{
* Corresponding author. Institute for Biotechnology and Bioengineering, Centre for Biological Engineering, University of Minho, Campus Gualtar, 4710-057 Braga, Portugal.

E-mail addresses: vsencadas@fisica.uminho.pt, vsencadas@ipca.pt (V. Sencadas).
}

regarding long term applications, such as drug delivery systems [6] or smart packaging [4,7], because the protein dissolves quickly in an aqueous environment. To overcome this limitation, cross-linking of gelatin is necessary.

Electrospinning allows the production of flexible and highly porous nanofiber structures by applying a high electric field to a droplet of polymer solution or melt $[8,9]$. Although gelatin has been successfully electrospun into fibers, the preparation of electrospun membranes raises some critical issues, such as the use of highly toxic solvents. Electrospun gelatin membranes can be obtained by the dissolution of the polymer in acetic acid/formic acid mixtures [10], 1,1,1,3,3,3-hexafluoro-2-propanol [11], 2,2,2-trifluoroethanol [12] and with acetic acid and ethyl acetate aqueous solutions [13].

Cross-linking of gelatin fiber membranes is a necessary step to increase its stability in aqueous environments. This can be achieved either using physical methods, such as 
dehydrothermal treatment and ultraviolet or gamma irradiation, or chemical methods which exploit a large number of chemical agents to modify gelatin functional side groups [14].

Gelatin chemical cross-linking can be achieved by glutaraldehyde (GA) [15,16], genipin [17,18] and EDC/Nhydroxysuccinimide (NHS) [19] in order to maintain morphological integrity of the as-spun membranes. GA is one of the most widely cross-linking agents used in polymeric materials from natural origin, such as gelatin [12] or chitosan [20]. It has been reported that cross-linking of gelatin microspheres with GA takes place more rapidly and is more efficient than with genipin. A maximum crosslinking degree of approximately $90 \%$ is achieved in less than $4 \mathrm{~h}$ of exposure to GA, whereas just around $60 \%$ of cross-linking was achieved after $72 \mathrm{~h}$ of exposure to genipin [21,22].

In vitro degradation of gelatin is usually performed in a PBS collagenase solution at $37{ }^{\circ} \mathrm{C}$. Rosellini et al. [23] studied the effect of the presence of collagenase in the phosphate buffer saline (PBS) solution during in vitro degradation of gelatin scaffolds cross-linked with GA, and found that a mainly hydrolytic degradation process occurs due to solvation and depolymerization of the polymeric chains [24].

Studies on the thermal properties of gelatin showed that it exhibits three main thermal degradation steps, the first being attributed to water desorption, and the others associated with protein degradation $[25,26]$. Gelatin thermal degradation kinetics has an activation energy $\left(E_{a c t}\right)$ ranging between 175 and $275 \mathrm{~kJ} \cdot \mathrm{mol}^{-1}$, the values increasing with the protein's molecular weight [25,27].

In the present study, electrospun gelatin membranes were prepared by electrospinning and cross-linked with GA in vapor phase in order to improve the fiber membranes stability in a moisture environment. Hydrolytic degradation was evaluated in PBS solution at $37^{\circ} \mathrm{C}$. Moreover, thermal degradation before and after GA crosslinking was studied. The main goal is to evaluate the potential of fish gelatin for biomedical applications.

\section{Experimental}

Electrospun membranes preparation: Fish gelatin was purchased from Sigma-Aldrich and dissolved in a blend of $\mathrm{N}, \mathrm{N}$-dimethylformamide (DMF, from Merck) and formic acid (FA, from Sigma-Aldrich) (40/60 vol/vol) to achieve a gelatin concentration of $30 \mathrm{wt} \%$ of the final solution. Fish gelatin was dissolved by stirring at $50{ }^{\circ} \mathrm{C}$ [28]. The polymer solution was then placed in a commercial plastic syringe $(10 \mathrm{~mL})$ fitted with a steel needle with $500 \mu \mathrm{m}$ inner diameter. Electrospinning was conducted at $1.5 \mathrm{kV} . \mathrm{cm}^{-1}$ with a high voltage power supply from Glassman (model PS/FC30P04). A syringe pump (from Syringepump) was used to feed the solutions into the needle tip at a rate of $0.2 \mathrm{~mL} \cdot \mathrm{h}^{-1}$, and the electrospun fibers were collected in a grounded collector.

Cross-linking: Samples were placed in a vapor chamber and then collected at different times, between 2 and $48 \mathrm{~h}$, with $20 \mathrm{~mL}$ of glutaraldehyde (GA, 50\% water, Panreac), which was vaporized at room temperature, placed at the bottom of the chamber.
Hydrolytic degradation: In vitro degradation of crosslinked gelatin electrospun membranes was carried out in PBS solution. The membrane samples were cut into squares of $25 \times 25 \mathrm{~mm}^{2}$ (triplicate samples were used for statistical purposes), immersed in $15 \mathrm{~mL}$ of PBS (pH 7.4; $0.8 \mathrm{~g} \mathrm{NaCl}$; $0.2 \mathrm{~g} \mathrm{KCl} ; 1.44 \mathrm{~g} \mathrm{Na}_{2} \mathrm{HPO}_{4} .2 \mathrm{H}_{2} \mathrm{O}$ and $0.2 \mathrm{~g} \mathrm{KH}_{2} \mathrm{PO}_{4}$ dissolved in $1 \mathrm{~L}$ of distilled water) and incubated in an air circulation oven (HERAEUS Vacuotherm) at $37^{\circ} \mathrm{C}$ for 15 days. The $\mathrm{pH}$ of the PBS solution was measured periodically and PBS was renewed every $72 \mathrm{~h}$. After specific periods of time, a membrane was removed from the PBS, washed with ultrapure water and dried in a vacuum oven (JP SELECTA Vacuotherm) at room temperature until constant mass was reached. Samples were weighed before and after degradation in an electronic quartz microbalance (M5P from Sartorius) with a resolution $\leq 0.001 \mathrm{mg}$. The extent of hydrolytic degradation $\left(W_{L}\right)$ was calculated by:

$W_{L}=\left(1-\frac{m_{s}}{m_{0}}\right)$

where, $m_{s}$ is the sample mass after an incubation period and $m_{0}$ is the initial sample mass.

Characterization: Electrospun fibers were coated with a thin gold layer using a sputter coater (Polaron, model SC502) and their morphology was analyzed using a scanning electron microscope (SEM, Quanta 650, from FEI) with an accelerating voltage of $15 \mathrm{kV}$. Nanofiber average diameter and the distribution were calculated over approximately 40 fibers using SEM images (5000× magnification) and Image $\mathrm{J}$ software. Differential scanning calorimetry measurements (DSC) were performed in a Perkin-Elmer Pyris-1 apparatus at a heating rate of $10{ }^{\circ} \mathrm{C} \cdot \mathrm{min}^{-1}$. Samples for the DSC studies were cut into small pieces from the middle region of the electrospun membranes and placed in $40 \mu \mathrm{L}$ aluminum pans. All experiments were performed under a nitrogen purge. The thermal degradation kinetics of the samples was characterized by thermogravimetric analysis (TGA) in a Perkin-Elmer Pyris-1 TGA apparatus at heating rate scans from $10{ }^{\circ} \mathrm{C} \cdot \mathrm{min}-1$ up to $40{ }^{\circ} \mathrm{C} \cdot \mathrm{min}^{-1}$ performed under a nitrogen atmosphere.

\section{Results and discussion}

The as-spun membranes (Fig. 1) show randomly oriented fibers with an average diameter of $240 \pm 58 \mathrm{~nm}$, with smooth surface and without bead defects (Fig. 1a). Since gelatin is a water soluble material, as-spun fiber membranes will partially or totally dissolve when in contact with an aqueous medium or high moisture environments [12]. To broaden the applications of these gelatin fibers to procedures requiring contact with water or biological medium gelatin fibers were exposed to a saturated atmosphere of GA in order to promote chemical cross-linking of the material. It was found that this chemical treatment does not influence the fiber average diameter for an exposure time up to $48 \mathrm{~h}$ [28]. Furthermore, the crosslinked fibers showed the same appearance as the as-spun ones (Fig. 1b). Similar behavior has been reported for other polymers such as chitosan [29].

Chemical cross-linking of gelatin with GA involves the reaction of $\varepsilon$-amino groups of Lys (lysine) or Arg (arginine) 


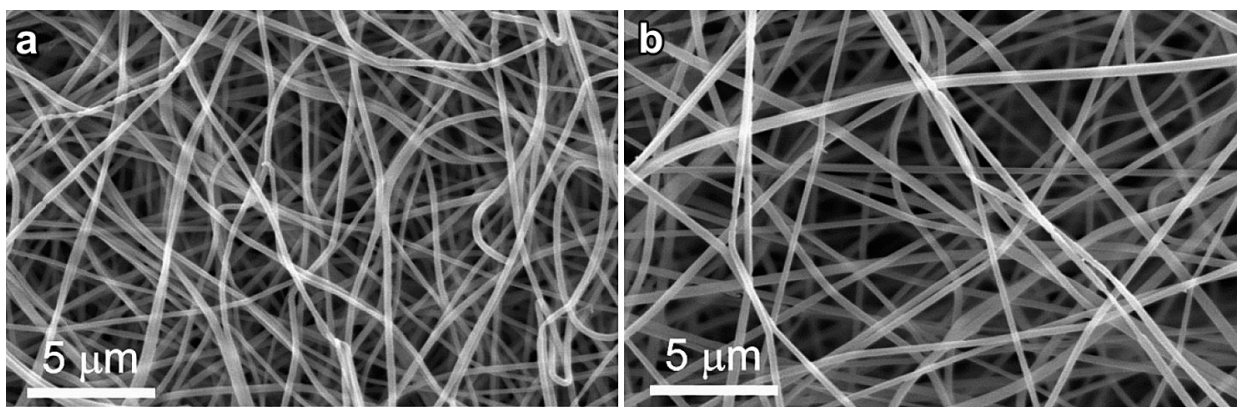

Fig. 1. Morphology of as-spun fish gelatin membranes: a) Before and b) After cross-linking with GA vapor phase during 48 h. samples were collected with an applied electric field of $1.25 \mathrm{kV} . \mathrm{cm}^{-1}$, a feed rate of $0.2 \mathrm{~mL} \cdot \mathrm{h}^{-1}$ and a needle inner diameter of $0.50 \mathrm{~mm}$.

of the polypeptide chains with aldehyde groups of the cross-linking agent by a Schiff base reaction [30]. Moreover, cross-linking is typically associated with a color change from white to pale yellow [12].

\subsection{Hydrolytic degradation}

Rosellini et al. [23] showed that in vitro degradation behavior at $37^{\circ} \mathrm{C}$ of gelatin cross-linked with GA films in PBS is quite similar to that obtained in PBS collagenase solution. Electrospun cross-linked samples were immersed in PBS solution and the extent of hydrolytic degradation was determined (Fig. 2). It was found that cross-linked electrospun gelatin fibers took less than $5 \mathrm{~min}$ to reach the equilibrium swelling state (data not shown) and can uptake as much as 14 times of its initial mass [28]. Gelatin weight loss occurred rapidly during the first week of incubation, losing around $50 \%$ of the sample initial mass (Fig. 2a) after approximately 9 days. After 15 days, a residual mass of $32 \%$ remained. Zhou et al. [31] suggested that gelatin mass loss during in vitro and in vivo degradation occurs primarily due to solvation and depolymerization of polymeric chains. It was observed also that electrospun cross-linked membranes still showed integrity after a week in vitro degradation (Fig. $2 \mathrm{~b}$ and $\mathrm{c}$ ).

\subsection{Thermal degradation}

Thermal degradation of gelatin occurs in three different stages (Fig. 3). The first stage occurs at temperatures from room temperature up to $200{ }^{\circ} \mathrm{C}$ with a mass loss of around $12 \%$. This is related to the loss of adsorbed water due to the handling of the sample at room conditions without any special care and, consequently, these data were not considered for the determination of the kinetic parameters $[25,32]$. Water bound to protein may be divided into three types according to its state: a) water bound by high-energy sorption centers that occurs inside collagen triple helix and which plays a major role in its stabilization by intramolecular bonds, the amount of water depending on the degree of helicity of the macromolecules. b) water absorbed by polar groups of gelatin and collagen macromolecules, bound to proteins by hydrogen bonds, located outside the helical fragments and also contributing to the stabilization of the collagen helical structure. The amount of this water in gelatin probably corresponds to the so-called monomolecular layer and can be considered as structural water. c) water absorbed by proteins to give polymolecular layers,

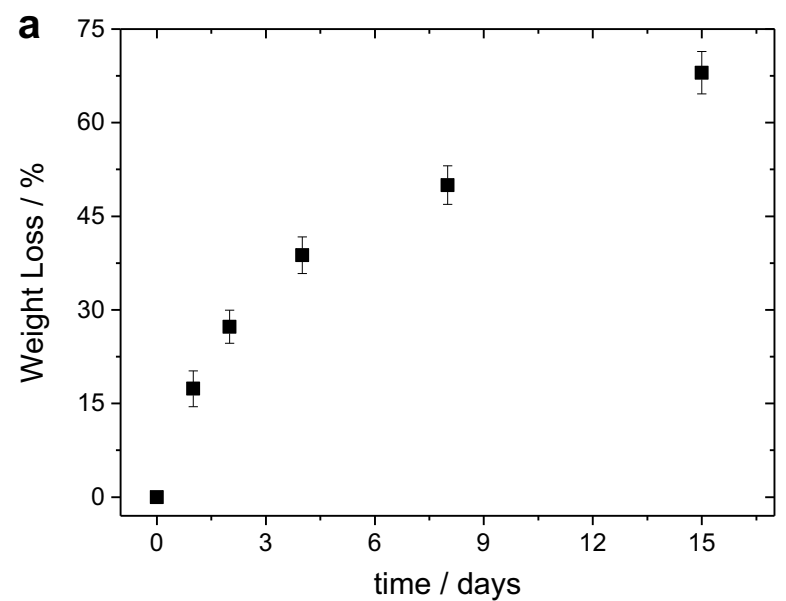

b
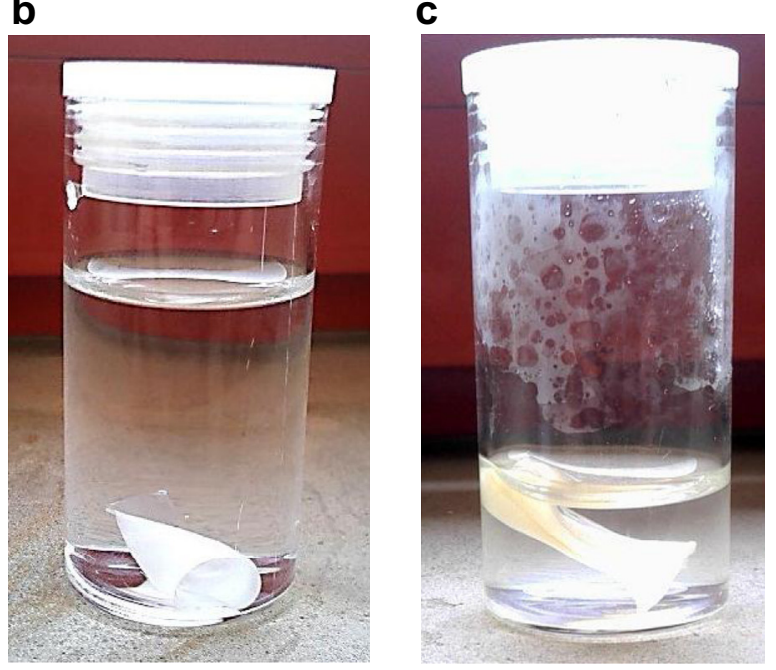

Fig. 2. a) Hydrolytic degradation kinetics for the electrospun gelatin, b) Gelatin membranes immersed in PBS for $1 \mathrm{~h}$ at $37^{\circ} \mathrm{C}$ and c) Gelatin membranes immersed in PBS for 7 days at $37^{\circ} \mathrm{C}$. 

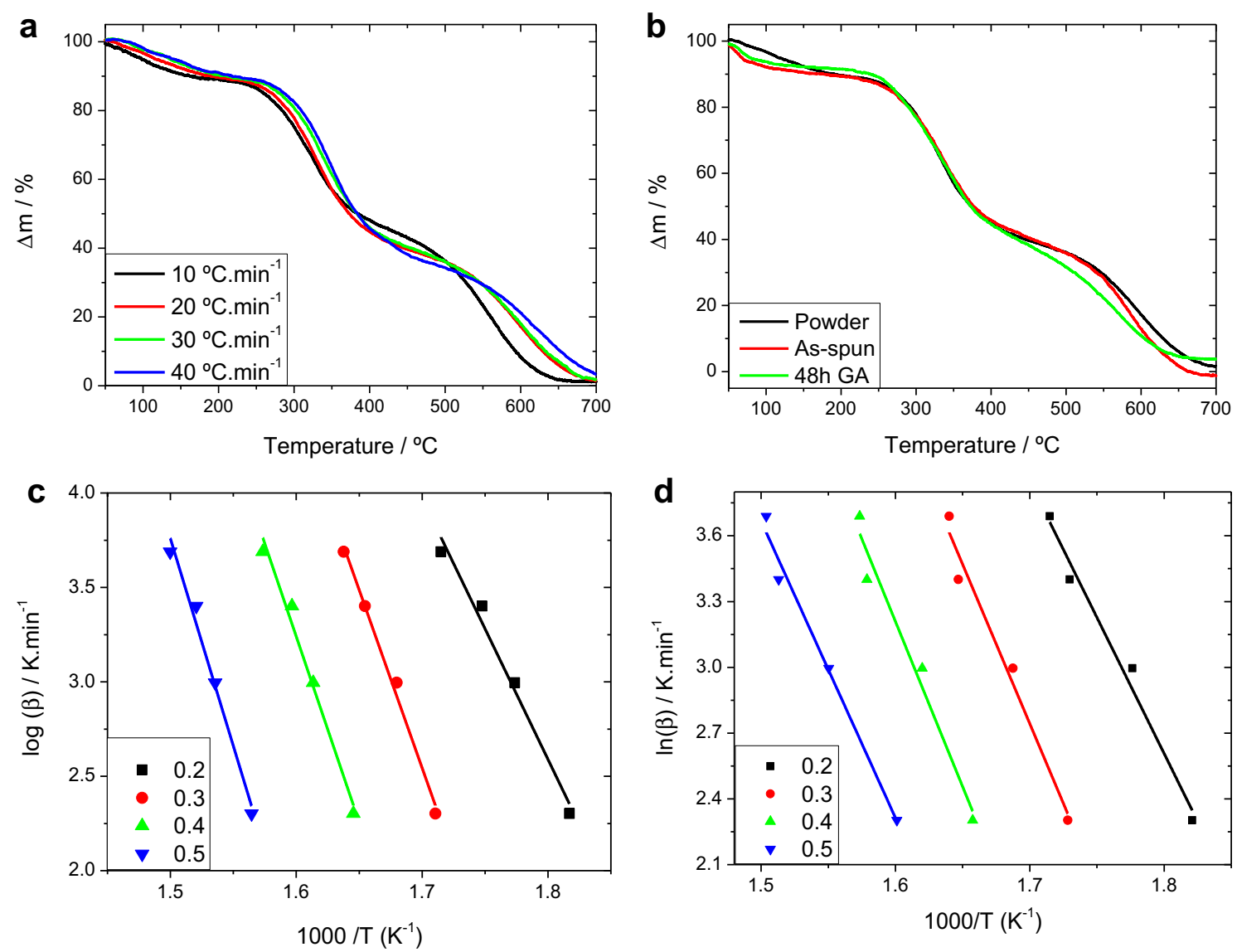

Fig. 3. TGA thermograms for a) gelatin powder, obtained at different heating rates; b) Electrospun membranes before and after different cross-linking periods, collected at a rate of $20^{\circ} \mathrm{C} \cdot \mathrm{min}^{-1}$; c) OFW fitting for fish gelatin powder and d) OFW fitting obtained for the gelatin fiber membranes cross-linked with GA during $48 \mathrm{~h}$ for different conversions.

consisting of the total amount of water bound in gelatin and the amount of structural water [33] (Fig. 4).

The second degradation stage occurs between 200 and $400{ }^{\circ} \mathrm{C}$ and is associated with protein degradation [25],

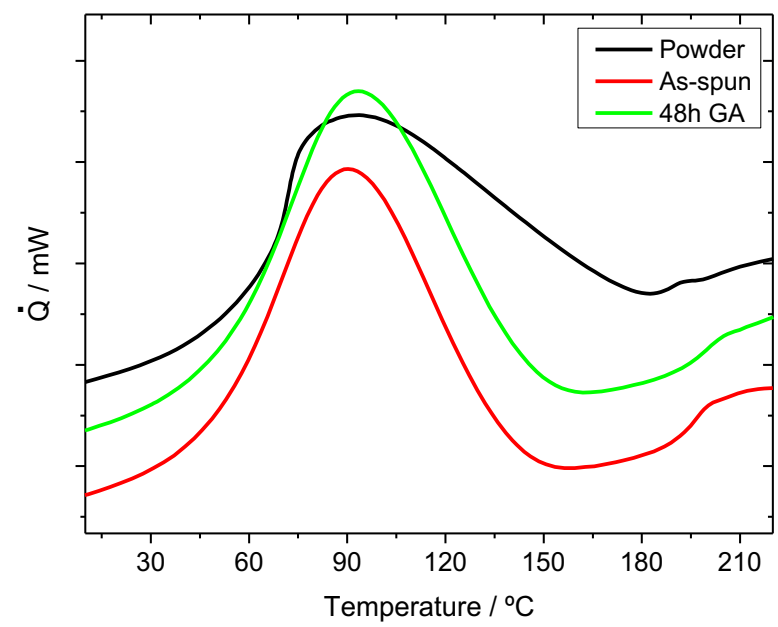

Fig. 4. DSC curves of gelatin raw material, as-spun and cross-linked with GA for $48 \mathrm{~h}$.
Finally, the third stage occurs between 400 and $600{ }^{\circ} \mathrm{C}$ and corresponds to the thermal decomposition of the gelatin networks (Fig. 3) [26]. In general, the thermal stability of the gelatin electrospun membranes is similar to that of the original powder (Fig. 3b).

The kinetics of the mass loss was studied through the analysis of experiments performed at different heating rates. As expected, increasing heating rates shift the onset temperature of the degradation processes to higher temperatures, not affecting any of the main characteristics of the process itself. A model for the decomposition kinetics has the following general form:

$\frac{\partial \alpha(t)}{\partial t}=k(T) f[\alpha(t)]$

where, $\alpha$ represents the degree of conversion of the sample under degradation, defined by:

$\alpha=\left(w_{0}-w_{t}\right) /\left(w_{0}-w_{\infty}\right)$

where $w_{0}, w_{t}$ and $w_{\infty}$ are the sample weights before degradation, at a given time $t$ and after complete degradation, respectively. The rate constant $k(t)$ changes with the absolute temperature according to the Arrhenius equation (Eq. (2)). $f(\alpha)$ represents the net result of elementary steps, 
as the polymer degradation is often a chain reaction. For the reaction model, $f(\alpha)=(1-\alpha)^{n}$ where $n$ is the reaction order assumed to remain constant during the degradation process.

The Ozawa-Flynn-Wall (OFW) [34,35] method assumes that the conversion function $f(\alpha)$ does not change with the heating rate for all values of the degree of conversion $\alpha$. This involves measuring the temperatures corresponding to fixed values of $\alpha$ from the experiments performed at different heating rates, $\beta$. In this theory:

$\ln (\beta)=\frac{\ln \left(A E_{a c t}\right)}{R}-\ln [f(\alpha)]-\frac{E_{a c t}}{R T}$

where $A$ is a pre-exponential factor $\left(\min ^{-1}\right), \mathrm{R}$ is the gas constant ( $\left.8.31 \mathrm{~J} \cdot \mathrm{mol}^{-1} \cdot \mathrm{K}^{-1}\right)$, and $E_{a c t}$ is the activation energy of the degradation process. By plotting $\ln (\beta)$ vs $1 / T$, the activation energy can be obtained, regardless of the reaction order of the system (Fig. $3 c$ and d). The validity of this model is based in the assumption that the conversion is constant for different heating rates[34,35].

The activation energy was calculated for the raw material and for the as-spun membranes before and after cross-linking with GA (Table 1). The values show that dissolution and processing conditions does not affect the thermal degradation of the material. It was previously reported that the [25] thermal activation energy for gelatin is between 175 and $275 \mathrm{~kJ} \mathrm{~mol}^{-1}$, the difference to the present investigation being attributed to the gelatin source, i.e. animal or fish gelatin, and molecular weight. After crosslinking, a decrease of $E_{a c t}$ was observed in both transition stages of the electrospun membranes (Table 1), which was probably due to random scission of polymer chains [25]. GA cross-linking of gelatin promotes water insoluble enzymes, cross-linking being associated with more rigid molecules with less ordered structures. Protein-protein interaction is, therefore, apparently affected by chemical cross-linking, and decreases the thermal stability of gelatin electrospun membranes. During heating, the initially ordered structure of the samples is gradually destroyed after breaking of inter- and intra-molecular hydrogen bonds, which are responsible for the maintenance of the polymeric chain [25,27].

Thermal properties of gelatin raw materials and electrospun membranes were also analysed by differential scanning calorimetry (DSC). DSC curves obtained for the different gelatin membranes showed similar trends, indicating that electrospinning and cross-linking with GA processes do not influence gelatin thermal properties. A broad endothermic peak was observed in the range of temperatures between 30 and $180{ }^{\circ} \mathrm{C}$, which is associated with the evaporation of bound water that was previously

Table 1

Evolution of $\mathrm{E}_{\mathrm{act}}$ for the fish gelatin powder and electrospun membranes before and after GA cross-linking.

\begin{tabular}{lll}
\hline Sample & $\mathrm{E}_{\mathrm{act}} / \mathrm{kJ} \cdot \mathrm{mol}^{-1}$ \\
\cline { 2 - 3 } & First-step & Second-step \\
\hline Powder & $175 \pm 27$ & $177 \pm 28$ \\
As-spun & $175 \pm 35$ & $189 \pm 35$ \\
48 h-GA & $110 \pm 13$ & $110 \pm 15$ \\
\hline
\end{tabular}

absorbed by the polymer membrane when in contact with air. TGA experiments showed that almost $12 \%$ of water can be absorbed by gelatin (Fig. 3) when in contact with air. Further, at $190^{\circ} \mathrm{C}$ an endothermic shoulder was observed, related to the helix-coil transition, i.e., the electrospinning process does not alter the formation of the characteristic helical structure between the gelatin chains [36].

\section{Conclusions}

Fish gelatin membranes were electrospun into random fiber membranes with an average diameter of $240 \pm 58 \mathrm{~nm}$. In order to improve stability in aqueous environments, chemical cross-linking with GA was performed for a $48 \mathrm{~h}$ period, and it was found that the cross-linking treatment does not influence the fiber average diameter of gelatin membranes.

Hydrolytic degradation of gelatin membranes was performed in a PBS solution at $37^{\circ} \mathrm{C}$, and after 15 days a weight loss of $68 \%$ was observed, attributed to solvation and depolymerization of the main polymer main chains. TGA studies of gelatin powder and electrospun membranes showed similar patterns and it was found that the electrospinning processing conditions do not affect the thermal degradation of the material. Further, a decrease of the activation energy was observed for the electrospun samples as compared to the raw protein. Cross-linking leads, therefore, to a more disordered structure and to the formation of polymer networks. Finally, it was found that electrospinning process does not influence the formation of the characteristic helical structure between the gelatin chains.

\section{Acknowledgements}

This work was supported by FEDER through the COMPETE Program and by the Portuguese Foundation for Science and Technology (FCT) in the framework of the Strategic Project PEST-C/FIS/UI607/2011 and by projects project references NANO/NMed-SD/0156/2007 and PTDC/ CTM-NAN/112574/2009. The authors also thank support from the COST Action MP1003, 2010 'European Scientific Network for Artificial Muscles'. DMC, JP and VS would like to acknowledge the FCT for the SFRH/BD/82411/2011, SFRH/BD/ $64901 / 2009$ and SFRH/BPD/64958/2009 grants respectively.

\section{References}

[1] B.-S. Chiou, R.J. Avena-Bustillos, P.J. Bechtel, H. Jafri, R. Narayan, S.H. Imam, G.M. Glenn, W.J. Orts, Cold water fish gelatin films: effects of cross-linking on thermal, mechanical, barrier, and biodegradation properties, European Polymer Journal 44 (2008) 3748-3753.

[2] H.W. Jones, R.A. Whitmore, in: U.S.P. Office (Ed.), Collagen Food Coating Composition and Method of Preparation, United States of America, (1972).

[3] A.A. Karim, R. Bhat, Fish gelatin: properties, challenges, and prospects as an alternative to mammalian gelatins, Food Hydrocolloids 23 (2009) 563-576.

[4] Y. Zhu, C. Gao, T. He, J. Shen, Endothelium regeneration on luminal surface of polyurethane vascular scaffold modified with diamine and covalently grafted with gelatin, Biomaterials 25 (2004) 423-430.

[5] M.C. Gómez-Guillén, M. Pérez-Mateos, J. Gómez-Estaca, E. LópezCaballero, B. Giménez, P. Montero, Fish gelatin: a renewable material for developing active biodegradable films, Trends in Food Science \& Technology 20 (2009) 3-16. 
[6] E. Esposito, R. Cortesi, C. Nastruzzi, Gelatin microspheres: influence of preparation parameters and thermal treatment on chemicophysical and biopharmaceutical properties, Biomaterials 17 (1996) 2009-2020.

[7] S. Farris, K.M. Schaich, L. Liu, P.H. Cooke, L. Piergiovanni, K.L. Yam, Gelatin-pectin composite films from polyion-complex hydrogels, Food Hydrocolloids 25 (2011) 61-70.

[8] J. Doshi, D.H. Reneker, Electrospinning process and applications of electrospun fibers, Journal of Electrostatics 35 (1995) 151-160.

[9] Seeram Ramakrishna, Kazutoshi Fujihara, Wee-Eong Teo, T.-C. Lim, Z. Ma, An Introduction to Electrospinning and Nanofibers, World Scientific Publishing Co. Pte. Ltd., Singapore, 2005.

[10] T. Balau Mindru, I. Balau Mindru, T. Malutan, V. Tura, Electrospinning of high concentration gelatin solutions, Journal of Optoelectronics and Advanced Materials 9 (2007) 3633-3638.

[11] M. Li, M.J. Mondrinos, M.R. Gandhi, F.K. Ko, A.S. Weiss, P.I. Lelkes, Electrospun protein fibers as matrices for tissue engineering, Biomaterials 26 (2005) 5999-6008.

[12] Y.Z. Zhang, J. Venugopal, Z.M. Huang, C.T. Lim, S. Ramakrishna, Crosslinking of the electrospun gelatin nanofibers, Polymer 47 (2006) 2911-2917.

[13] J.-H. Song, H.-E. Kim, H.-W. Kim, Production of electrospun gelatin nanofiber by water-based co-solvent approach, J Mater Sci: Mater Med 19 (2008) 95-102.

[14] S. Panzavolta, M. Gioffrè, M.L. Focarete, C. Gualandi, L. Foroni, A. Bigi, Electrospun gelatin nanofibers: optimization of genipin crosslinking to preserve fiber morphology after exposure to water, Acta Biomaterialia 7 (2011) 1702-1709.

[15] M. Skotak, S. Noriega, G. Larsen, A. Subramanian, Electrospun crosslinked gelatin fibers with controlled diameter: the effect of matrix stiffness on proliferative and biosynthetic activity of chondrocytes cultured in vitro, Journal of Biomedical Materials Research - Part A 95 (2010) 828-836

[16] M. Li, X. Liu, Comparison of characteristics between glutaraldehydeand genipin-crosslinked gelatin microspheres, Chinese Journal of Reparative and Reconstructive Surgery 23 (2009) 87-91.

[17] Y. Su, X. Mo, Genipin crosslinked gelatin nanofibers for tissue engineering, Journal of Controlled Release: Official Journal of the Controlled Release Society 152 (Suppl 1) (2011) e230-e232.

[18] K. Sisson, C. Zhang, M.C. Farach-Carson, D.B. Chase, J.F. Rabolt, Evaluation of cross-linking methods for electrospun gelatin on cell growth and viability, Biomacromolecules 10 (2009) 1675-1680.

[19] J.Y. Lai, Porous gelatin hydrogel carriers: evaluation of cross-linking degree for optimal cell delivery performance (2011), pp. 273-281.

[20] V. Sencadas, D.M. Correia, C. Ribeiro, S. Moreira, G. Botelho, J.L. Gómez Ribelles, S. Lanceros-Mendez, Physical-chemical properties of cross-linked chitosan electrospun fiber mats, Polymer Testing 31 (2012) 1062-1069.

[21] H.-C. Liang, W.-H. Chang, K.-J. Lin, H.-W. Sung, Genipin-crosslinked gelatin microspheres as a drug carrier for intramuscular administration: In vitro and in vivo studies, Journal of Biomedical Materials Research Part A 65A (2003) 271-282.
[22] S.-C. Wu, W.-H. Chang, G.-C. Dong, K.-Y, Chen, Y.-S. Chen, C.-H. Yao, Cell adhesion and proliferation enhancement by gelatin nanofiber scaffolds, Journal of Bioactive and Compatible Polymers 26 (2011) 565-577.

[23] E. Rosellini, C. Cristallini, N. Barbani, G. Vozzi, P. Giusti, Preparation and characterization of alginate/gelatin blend films for cardiac tissue engineering, Journal of Biomedical Materials Research Part A 91A (2009) 447-453.

[24] S. Gorgieva, V. Kokol, Collagen- vs. gelatine-based biomaterials and their bocompatibility: review and perspectives, in: P.R. Pignatello (Ed.), Biomaterials Applications for Nanomedicine, InTech, 2011.

[25] P.L.M. Barreto, A.T.N. Pires, V. Soldi, Thermal degradation of edible films based on milk proteins and gelatin in inert atmosphere, Polymer Degradation and Stability 79 (2003) 147-152.

[26] R. Mishra, A. Majeed, A. Banthia, Development and characterization of pectin/gelatin hydrogel membranes for wound dressing, International Journal of Plastics Technology 15 (2011) 82-95.

[27] A. Kamińska, A. Sionkowska, The effect of UV radiation on the values of thermal parameters of collagen containing $\beta$-carotene, Polymer Degradation and Stability 65 (1999) 87-90.

[28] Jorge Padrão, João P. Silva, Ligia Rodrigues, F. Dourado, Miguel Gama, S. Lanceros-Méndez, V. Sencadas, Modifying fish gelatin electrospun membranes for biomedical applications: cross linking and swelling behaviour, International Journal of Biological Macromolecules (2013). Submitted.

[29] V. Sencadas, C. Ribeiro, J. Nunes-Pereira, V. Correia, S. LancerosMéndez, Fiber average size and distribution dependence on the electrospinning parameters of poly(vinylidene fluoride-trifluoroethylene) membranes for biomedical applications, Applied Physics A: Materials Science \& Processing 109 (2012) 685-691.

[30] S. Gorgieva, V. Koko, Collagen- vs. gelatine-based biomaterials and their biocompatibility: review and perspectives, in: R. Pignatello (Ed.), Biomaterials Applications for Nanomedicine, InTech, 2011.

[31] Z.H. Zhou, S.L. He, T.L. Huang, L.H. Liu, Q.Q. Liu, Y.M. Zhao, B.L. Ou, W.N. Zeng, Z.M. Yang, D.F. Cao, Degradation behaviour and biological properties of gelatin/hyaluronic acid composite scaffolds, Materials Research Innovations 0 (2013) 1-5.

[32] M. Sadeghi, B. Heidari, Crosslinked graft copolymer of methacrylic acid and gelatin as a novel hydrogel with pH-responsiveness properties, Materials 4 (2011) 543-552.

[33] P.V. Kozlov, G.I. Burdygina, The structure and properties of solid gelatin and the principles of their modification, Polymer 24 (1983) 651-666

[34] J.H. Flynn, L.A. Wall, A quick, direct method for the determination of activation energy from thermogravimetric data, Journal of Polymer Science Part B: Polymer Letters 4 (1966) 323-328.

[35] T. Ozawa, A new method to analyze thermogravimetric data, Bulletin of the Chemical Society of Japan 38 (1965) 1881-1991.

[36] C. Peña, K. de la Caba, A. Eceiza, R. Ruseckaite, I. Mondragon, Enhancing water repellence and mechanical properties of gelatin films by tannin addition, Bioresource Technology 101 (2010) 6836-6842. 